\title{
AN ITERATIVE METHOD FOR SOLUTION OF NONLINEAR OPERATOR EQUATION
}

\author{
NGUYEN BUONG
}

\begin{abstract}
In the note, for finding a solution of nonlinear operator equation of Hammerstein's type an iterative process in infinite-dimensional Hilbert space is shown, where a new iteration is constructed basing on two last steps. An example in the theory of nonlinear integral equations is given for illustration.
\end{abstract}

\section{INTRODUCTION}

Let $H$ be a real Hilbert space with the norm and scalar product denoted by \|\| and $\langle.,$.$\rangle , respectively.$

Let $F_{i}, i=1,2$, be nonlinear monotone operators in $H$, i.e.

$$
\left\langle F_{i}(x)-F_{i}(y), x-y\right\rangle \geq 0, \quad \forall x, y \in D\left(F_{i}\right) \equiv H, i=1,2 .
$$

The operator equation of Hammerstein's type

$$
x+F_{2} F_{1}(x)=f_{0}, \quad f_{0} \in H
$$

was considered by several authors (see [1], [2], [4-7], [12-17] and bibliography there). In [10], an iterative process was given for solving (1.1) with the linear property of $F_{2}$. In [6], the author proposed an method of regularization for the solution of (1.1) in the case, where both the operators $F_{i}$ are nonlinear and monotone.

In the note, basing on our result in [6] and the idea of iterative regularization proposed by A. Bakyshinski (see [3]), we give a two-step iteration method for solving (1.1) in infinite-dimensional Hilbert space $H$. The result is illustrated by an example in the theory of nonlinear integral equations.

Note that, recently, the problem of approximating a solution of $(1.1)$ is investigated extensively because of its importance in applications (see [8], [9], [11], $[16])$. 


\section{MAIN RESULT}

Let $x^{1}$ and $x^{2}$ be two arbitrary elements of $H$. The iteration procedure is defined by

$$
\begin{gathered}
x^{n+2}=\varphi_{1}^{n+1}\left(x^{n+1}\right)+\beta_{n+1}\left[\varphi_{2}^{n}\left(\left(x^{n+1}-\varphi_{1}^{n}\left(x^{n}\right)\right) / \beta_{n}\right)-\beta_{n} x^{n}\right] \\
n=1,2, \ldots
\end{gathered}
$$

where

$$
\begin{gathered}
\varphi_{i}^{n}(x)=x-\beta_{n}\left(F_{i}(x)+\alpha_{n} x+a_{i} f_{0}\right), i=1,2, \\
a_{1}=0, \quad a_{2}=-1,
\end{gathered}
$$

and $\left\{\alpha_{n}\right\}$ and $\left\{\beta_{n}\right\}$ are two sequences of positive numbers. Later, we see, as in [3], that $\alpha_{n}$ plays the role of regularization and $\beta_{n}$, the role of iteration parameter.

Theorem. If (1.1) has a solution and there exist the constants $L_{i}>0$ such that

$$
\left\|F_{i}(x)\right\| \leq L_{i}(1+\|x\|), i=1,2, \forall x \in H,
$$

then iteration process (2.1) converges to a solution of (1.1) under the condition

$$
\alpha_{n}, \beta_{n}>0, \quad \alpha_{n} \rightarrow 0, \quad \lim \frac{\left|\beta_{n}-\beta_{n+1}\right|}{\beta_{n} \alpha_{n}^{2}}=0, \quad \sum_{1}^{\infty} \alpha_{n} \beta_{n}=\infty .
$$

Proof. Put

$$
y^{n}=\left(x^{n+1}-\varphi_{1}^{n}\left(x^{n}\right)\right) / \beta_{n}, \quad n=1,2, \ldots
$$

Then from (2.1) and (2.2) we have

$$
\begin{aligned}
y^{n+1} & =\left(x^{n+2}-\varphi_{1}^{n+1}\left(x^{n+1}\right)\right) / \beta_{n+1} \\
& =\varphi_{2}^{n}\left(y^{n}\right)-\beta_{n} x^{n} \\
& =y^{n}-\beta_{n}\left(F_{2}\left(y^{n}\right)+x_{n}+\alpha_{n} y^{n}-f_{0}\right) .
\end{aligned}
$$

On the other hand, from (2.3) and (2.2) we also obtain

$$
\begin{aligned}
x^{n+1} & =\varphi_{1}^{n}\left(x^{n}\right)+\beta_{n} y^{n} \\
& =x^{n}-\beta_{n}\left(F_{1}\left(x^{n}\right)-y_{n}+\alpha_{n} x^{n}\right), \quad n=1,2, \ldots
\end{aligned}
$$


In the Hilbert space $H_{1}=H \times H$ with the scalar product denoted by $\left\langle z_{1}, z_{2}\right\rangle_{1}=$ $\left\langle x_{1}, x_{2}\right\rangle+\left\langle y_{1}, y_{2}\right\rangle$, where $z_{i}=\left[x_{i}, y_{i}\right], x_{i}, y_{i} \in H$, we can write

$$
\begin{aligned}
z^{n+1} & =z^{n}-\beta_{n}\left(\xi\left(z^{n}\right)+\alpha_{n} z^{n}-\bar{f}_{0}\right), \\
\mathcal{F}\left(z^{n}\right) & =\left[F_{1}\left(x^{n}\right), F_{2}\left(y^{n}\right)\right]+\left[-y^{n}, x^{n}\right], \\
z^{n} & =\left[x^{n}, y^{n}\right], \bar{f}_{0}=\left[\theta, f_{0}\right],
\end{aligned}
$$

where the $\theta$ denotes the zero element in $H$. It is easy to verify that in the Hilbert space $H_{1}, \mathcal{F}$ is a monotone operator. However, without any difficulty we can see that $\mathcal{I}$ satisfies the condition

$$
\|\mathcal{F}(z)\| \leq \sqrt{2} \max L_{i}\left(1+\|z\|_{1}\right),
$$

where $\|\cdot\|_{1}$ is the norm of $H_{1}$ generated by $\langle., .\rangle_{1}$.

Applying Theorem 5.1 (p. 144) in [3] to the process (2.4), we can conclude that the sequence $\left\{z^{n}\right\}$ converges in $H_{1}$ to $z_{0}=\left[x_{0}, F_{1}\left(x_{0}\right)\right]$, one solution of the equation

$$
\mathcal{F}(z)=\bar{f}_{0} .
$$

Therefore, the sequence $\left\{x^{n}\right\}$ converges in $H$ to $x_{0}$, as $n \rightarrow \infty$. Theorem is proved.

Remarks. 1. The sequence $\beta_{n}=(1+n)^{-1 / 2}$ and $\alpha_{n}=(1+n)^{-p}, 0<p<1 / 2$, satisfy all the conditions in the theorem.

2. If $F_{i}$ are Lipschitz continuous with a Lipschitz constants $L_{i}$, then $₹$ also is Lipschitz continuous with Lipschitz constant $\mathcal{L}=2 \sqrt{\max \left\{1, L_{1}, L_{2}\right\}}$. Applying Theorem 5.2 in [3], we obtain the result that the iteration process $(2.1)$ converges in $H$ to a solution of (1.1), if

$$
\varlimsup_{n \rightarrow \infty} \beta_{n} \frac{\left(1+\alpha_{n}^{2}\right)}{\alpha_{n}}<\frac{2}{\mathcal{L}^{2}} .
$$

In this case, we can chose the sequence $\beta_{n}=\theta \alpha_{n}$,

$$
\alpha_{n}=(1+n)^{-p}, \quad 0<p<1 / 2, \quad \theta<\frac{2}{\left(1+\alpha_{0}\right)^{2} \mathcal{L}^{2}} .
$$

\section{APPLICATION}

Consider the nonlinear integral equation of Hammerstein's type

$$
\varphi(t)+\int_{0,}^{1} k(t, s) f(\varphi(s)) d s=f_{0}(t), \quad t \in[0,1], \quad \varphi \in L_{2}[0,1],
$$


where $f_{0}(t) \in L_{2}[0,1], k(t, s) \geq 0$ is continuous and $f(t)$ is a nondecreasing and bounded function satisfying the condition $|f(t)| \leq a_{0}+b_{0}|t|, t \in R$. Then,

$$
\begin{aligned}
& \left(F_{1} \varphi\right)(t)=f(\varphi(t)), \varphi(t) \in L_{2}[0,1] \\
& \left(F_{1} \xi\right)(t)=\int_{0}^{1} k(t, s) \xi(s) d s, \xi(t) \in L_{2}[0,1]
\end{aligned}
$$

Since $k(t, s) \geq 0$ and $f(t)$ is nondeacreasing, then $F_{i}, i=1,2$, are monotone. The continuity of $k(t, s)$ implies that $F_{2}$ is bounded. It is not difficult to prove that $F_{i}$ satisfy the conditions of the main theorem. Therefore, in order to obtain approximate solution for (3.1) we can apply the process (2.1) with defined above $F_{i}$ and $\alpha_{n}, \beta_{n}$ in the remark 1 .

\section{REFERENCES}

1. H. Amann, Ein Existenz und Eindertigkeitssatz fur die Hammersteinshe Gleichung in Banachraumen, Math. Z., 111 (1969), 175 - 190.

2. H. Amann, Uber die naherungsweise Losung nichlinearer Integralgleichungen, Numer. Math., 19 (1972), $29-45$.

3. A. Bakushinsky and A. Goncharsky, $\mathrm{Il}$-posed problems: Theory and applications, (Kluwer Academic Publishers) 1994.

4. H. Brezis and F. Browder, Nonlinear Integral Equations and Systems of Hammerstein's Type, Adv. Math. 10 (1975), $115-144$.

5. F. Browder, Nonlinear Functional Analysis and Nonlinear Equations of Hammerstein and Uryshon Type, in: E.H. Zarantonello, ed., Contribution to Nonlinear Functional Analysis, Academic Press, New - York, 1971, 425-500.

6. N. Buong, On solutions of the equations of Hammerstein type in Banach spaces, J. of Math. Computation \& Math. physics USSR, 25, No. 8 (1985), 1256 - 1280 (Russian).

7. C. Dolph and G. Minty, On Nonlinear Integral Equations of Hammerstein Type, in: P. Anselon, ed., Nonlinear Integral Equations, Wis. Press, Nadison, 1964, 99 - 154 .

8. H. Kaneko, R. Noren, and Y. Xu, Numerical solution for weakly singular Hammerstein equations and their superconvergence, J. Int. Eq. Appl., 4 (1992), $391-407$.

9. H. Kaneko and Y. Xu, Degenerate kernel method for Hammerstein equations, Math. Comp., 56 (1991), $141-148$.

10. R. Kannan and H. Salehi, Random nonlinear equations and monotonic nonlinearities, J. Math. Anal. Appl., 78 (1980), 488 - 496.

11. S. Kumar, Superconvergence of a Collocation-type Method for Hammerstein Equations, IMA Journal of Numerical Analysis, 7 (1987), 313 - 325.

12. C. D. Panchal, Existence Theorems for Equations of Hammerstein Type, The Quart. J. of Math., 35 (1984), $311-320$.

13. D. Pascali and S. Sburlan, Nonlinear mappings of monotone type, Bucur. Roumania, 1978.

14. W. V. Petryshyn and R. M. Fitzpatrick, New existence theorems for nonlinear equation of Hammerstein type, Thans. AMS, 160 (1971), 39-63. 
15. L. Tartar, Topics in Nonlinear Analysis, Publ. d'Orsey, 1978.

16. D. Vaclav, Monotone Operators and Applications in Control and Network Theory, Ams. - Oxf. - Newyork, Elsevier, 1979.

17. M. M. Vainberg, Variational method and method of monotone operators, Moscow, Nauka, 1972 (Russian).

Institute of Information Technology

National Centre for Science and Technology of Vietnam.

Received: October 20, 1996 